\title{
Glutathione Peroxidase Potentiates the Inhibition of Platelet Function by S-Nitrosothiols
}

\author{
Jane E. Freedman, Balz Frei, George N. Welch, and Joseph Loscalzo \\ Whitaker Cardiovascular Institute and Evans Department of Medicine, Boston University School of Medicine, Boston, \\ Massachusetts 02118
}

\begin{abstract}
GSH peroxidase ( $\mathrm{Px})$ catalyzes the reduction of lipid hydroperoxides (LOOH), known metabolic products of platelets and vascular cells. Because interactions between these cells are modulated by nitric oxide (NO) and LOOH inactivate NO, we investigated the effect of GSH-Px on the inhibition of platelet function by the naturally occurring S-nitrosothiol, S-nitroso-glutathione (SNO-Glu). Concentrations of SNO-Glu that alone did not inhibit platelet function (subthreshold inhibitory concentrations) were added to plateletrich plasma together with GSH-Px $(0.2-20 \mathrm{U} / \mathrm{ml})$; this led to a dose-dependent inhibition of platelet aggregation with an $\mathrm{IC}_{50}$ of $0.6 \mathrm{U} / \mathrm{ml} \mathrm{GSH-Px}$. In the presence of subthreshold inhibitory concentrations of SNO-Glu, the LOOH, 5-hydroperoxy-6,8,11,14-eicosatetraenoic acid, increased platelet aggregation, an effect reversed by GSH-Px. Glutathione and SNO-Glu were equally effective as cosubstrates for GSHPx. Incubation of SNO-Glu with GSH-Px for $1 \mathrm{~min}$ led to a $48.5 \%$ decrease in the concentration of SNO-Glu. Incubation of SNO-Glu with serum albumin led to the formation of S-nitroso-albumin, an effect enhanced by GSH-Px. These observations suggest that GSH-Px has two functions: reduction of $\mathrm{LOOH}$, thereby preventing inactivation of NO, and metabolism of SNO-Glu, thereby liberating NO and/or supporting further transnitrosation reactions. ( $J$. Clin. Invest. 1995. 96:394-400.) Key words: nitric oxide $\bullet$ S-nitrosothiols - endothelium-derived relaxing factor • lipid peroxides • glutathione peroxidase
\end{abstract}

\section{Introduction}

An important metabolic product of the endothelial cell is endothelium-derived relaxing factor (EDRF), ${ }^{1}$ a known endogenous

This work was presented in part at the 67th Scientific Session of the American Heart Association, Dallas, TX, November 14-17, 1994.

Address correspondence to Joseph Loscalzo, Whitaker Cardiovascular Institute, Center for Advanced Biomedical Research, Boston University School of Medicine, 700 Albany Street, Boston, MA 02118. Phone: 617-638-4890; FAX: 617-638-4066.

Received for publication 13 October 1994 and accepted in revised form 17 March 1995.

1. Abbreviations used in this paper: 12(S)HETE, 12-hydroxy5,8,10,14-eicosatetraenoic acid; $12(\mathrm{~S}) \mathrm{HpETE}, 12$-hydroperoxy5,8,10,14-eicosatetraenoic acid; EDRF, endothelium-derived relaxing factor; $\mathrm{LOOH}$, lipid hydroperoxide; NO, nitric oxide; Px, peroxidase; SNO-Glu, S-nitroso-glutathione; SNO-Cys, S-nitroso-L-cysteine.

J. Clin. Invest.

(C) The American Society for Clinical Investigation, Inc.

0021-9738/95/07/0394/07 \$2.00

Volume 96, July 1995, 394-400 vasodilator. EDRF also inhibits platelet aggregation $(1,2)$ and prevents adhesion of platelets to the endothelium (3). EDRF exhibits properties of both nitric oxide (NO) and the naturally occurring thiol adducts of NO, S-nitrosothiols, such as inhibiting platelet function and elevating intracellular levels of cGMP (4). These alterations in cGMP levels may represent a negative feedback pathway in the regulation of platelet activation. In vivo, endogenously synthesized NO has been shown to prevent thrombosis in a model of endotoxin-induced glomerular damage (5), as well as attenuate platelet adhesion to damaged endothelium (6).

In the setting of inflammation, oxygen-derived free radicals can promote lipid peroxidation leading to the generation of lipid alkoxyl and peroxyl radicals. Interestingly, NO itself has antioxidant effects as a consequence of its direct reaction with alkoxyl and peroxyl radical intermediates generated during lipid peroxidation, thus terminating lipid radical chain propagation reactions and leading to the formation of lipid peroxynitrites (7). Such interactions may be important in vivo as cell and tissue damage can occur secondary to oxidation of lipids.

The antioxidant enzyme GSH peroxidase (Px) reduces lipid hydroperoxides $(\mathrm{LOOH})$ to the corresponding alcohols by the oxidation of reduced GSH (8). Activation and aggregation of platelets result in the release of membrane-bound stores of arachidonic acid, which, via the lipoxygenase pathway, are converted to a variety of biologically active eicosanoids, including LOOH. These LOOHs, such as 12-hydroperoxy-5,8,10,14-eicosatetraenoic acid (12(S)HpETE), can be further metabolized by GSH-Px to the corresponding alcohol or lipid hydroperoxide, e.g., 12-hydroxy-5,8,10,14-eicosatetraenoic acid (12(S)HETE). When added exogenously, HpETEs are potent inhibitors of platelet aggregation (9).

Essentially all of the hydroperoxide-reducing activity in plasma is attributable to GSH-Px (10). Patients with coronary artery disease have lower plasma and platelet GSH-Px levels and increased plasma levels of malondialdehyde, a LOOH decomposition product (11). In addition, platelet aggregability is increased in patients with coronary artery disease. In the absence of the trace element selenium, plasma GSH-Px protein and its activity are deficient (8), and low selenium intake is associated with an increased risk of coronary artery disease (12). A decreased incidence of atherosclerotic cardiovascular disease is found among populations with a high intake of fish, which, in addition to having high levels of omega- 3 polyunsaturated fatty acids, also contains significant amounts of selenium (13).

GSH-Px activity appears to be decreased in coronary artery disease, a condition also associated with impaired release of NO and increased thrombosis. Thus, in this study we investigated possible effects of GSH-Px on NO and S-nitrosothiol metabolism and the consequences of these effects on platelet function. We demonstrate that GSH-Px potentiates NO-mediated platelet inhibition and conclude, therefore, that GSH-Px may be important in the regulation of EDRF action. 


\section{Methods}

Chemicals and solutions. $\mathrm{H}_{2} \mathrm{O}_{2}$, GSH, GSH reductase (baker's yeast), GSH-Px (human or bovine erythrocyte), SOD (bovine erythrocyte), catalase (bovine liver), reduced NADPH, L-cysteinyl-glycine, D,L-homocysteine, zinc chloride, $\beta$-mercaptosuccinic acid, L-cysteine, sodium arachidonate, sodium nitrite, sulfanilamide, BSA, hydrochloric acid, and TCA were purchased from Sigma Chemical Co. (St. Louis, MO). $\mathrm{N}$ (1-naphthyl)ethylene-diamine dihydrochloride was purchased from Aldrich Chemical Co. (Milwaukee, WI). 5-Hydroperoxy-6,8,11,14-eicosatetraenoic acid (5(S)-HpETE), 5-hydroxy-6,8,11,14-eicosatetraenoic acid (5(S)-HETE), 12(S)HpETE, and 12(S)-HETE (all [S$(\mathrm{E}, \mathrm{Z}, \mathrm{Z}, \mathrm{Z})]$ configuration ), as well as cGMP enzyme immunoassay kits, were purchased from Cayman Chemical Co. (Ann Arbor, MI). PBS, $\mathrm{pH} 7.4$, consisted of $10 \mathrm{mM}$ sodium phosphate and $150 \mathrm{mM} \mathrm{NaCl}$ and was chelex-treated to remove contaminating transition metals.

Preparation of platelet-rich plasma. Venous blood, anticoagulated with $89.4 \mathrm{mM}$ sodium citrate, $15.6 \mathrm{mM}$ citric acid, $16.1 \mathrm{mM} \mathrm{NaH}_{2} \mathrm{PO}_{4}$, and $142 \mathrm{mM}$ dextrose, was obtained from healthy volunteer donors who had not ingested platelet inhibitors for $\geq 10 \mathrm{~d}$. Platelet-rich plasma (PRP) was prepared by centrifugation at $150 \mathrm{~g}$ for $10 \mathrm{~min}$ at $25^{\circ} \mathrm{C}$. Platelet-poor plasma was prepared by centrifugation at $800 \mathrm{~g}$ for 10 min. Platelets were adjusted to $2 \times 10^{8}$ platelets $/ \mathrm{ml}$ after counts were determined using a Coulter Counter (model ZM, Coulter Electronics, Hialeah, FL).

Platelet aggregation: incubations with S-nitrosothiols, antioxidant enzymes, and lipid hydroperoxides. Aggregation was induced by adding $500 \mu \mathrm{M}$ arachidonate to platelets. Aggregation studies were performed at $37^{\circ} \mathrm{C}$ with a constant stirring rate of $1200 \mathrm{rpm}$ in a BioData fourchamber aggregometer (Bio/Data Corp., Horsham, PA). Aggregation was quantified by measuring the maximal extent of change in light transmittance (14).

S-nitrosothiols were prepared immediately before use by mixing equimolar concentrations of $\mathrm{NaNO}_{2}$ and thiol (GSH or L-cysteine) in $0.1 \mathrm{~N} \mathrm{HCl}$ on ice (15). Further dilutions were made in PBS at room temperature. Threshold concentrations (inhibitory or aggregatory) of S-nitroso-glutathione (SNO-Glu), S-nitroso-L-cysteine (SNO-Cys), or nitroprusside were determined immediately before the aggregation ex periment for which they were needed and repeated with each set of aggregation experiments. In some experiments platelets were incubated with GSH-Px, SOD, catalase, LOOH, or a GSH-Px inhibitor before incubation with S-nitrosothiol. Each incubation was conducted for $30 \mathrm{~s}$ with stirring at $37^{\circ} \mathrm{C}$. The S-nitrosothiol was then added and stirred for $30 \mathrm{~s}$ at $37^{\circ} \mathrm{C}$ immediately before addition of the platelet agonist for the given experiment.

Human or bovine erythrocyte GSH-Px preparations were used in these studies. Despite some known differences, both the plasma and erythrocyte forms have the same substrate specificity and $K_{\mathrm{m}}$ for GSH (16), this being the probable rate-limiting step in the platelet aggregation experiments (17).

Cyclic nucleotide assay. After completion of the previously described incubations, TCA (final concentration, $5 \% \mathrm{vol} / \mathrm{vol}$ ) was added to PRP. Samples were vortexed, placed on ice, and centrifuged at 1,500 $g$ for $10 \mathrm{~min}$ at $4^{\circ} \mathrm{C}$. The supernatant was extracted with diethyl ether and assayed for cGMP by an ELISA methodology using cGMP antiserum (Cayman Chemical Co.).

Measurement of S-nitroso-albumin. SNO-Glu was incubated with serum albumin and $\mathrm{H}_{2} \mathrm{O}_{2}$ in PBS, aliquots of the reaction solution were removed over time, and the aliquots were mixed either with hydrochloric acid or with perchloric acid (to precipitate protein). This incubation was also repeated in the presence of $5 \mathrm{U} / \mathrm{ml}$ GSH-Px. Total nonprecipitable S-nitrosothiol (SNO-Glu) and precipitable S-nitrosothiol (S-nitrosoalbumin) were determined by the method of Saville (18). This experiment was also conducted incubating freshly prepared S-nitroso-albumin (19), GSH, and $\mathrm{H}_{2} \mathrm{O}_{2}$ in the presence or absence of $5 \mathrm{U} / \mathrm{ml} \mathrm{GSH}-\mathrm{Px}$.

Determination of GSH-Px activity. Endogenous plasma GSH-Px or purified enzyme activity was assayed by coupling the peroxidase reaction with the reduction of oxidized glutathione by glutathione reductase

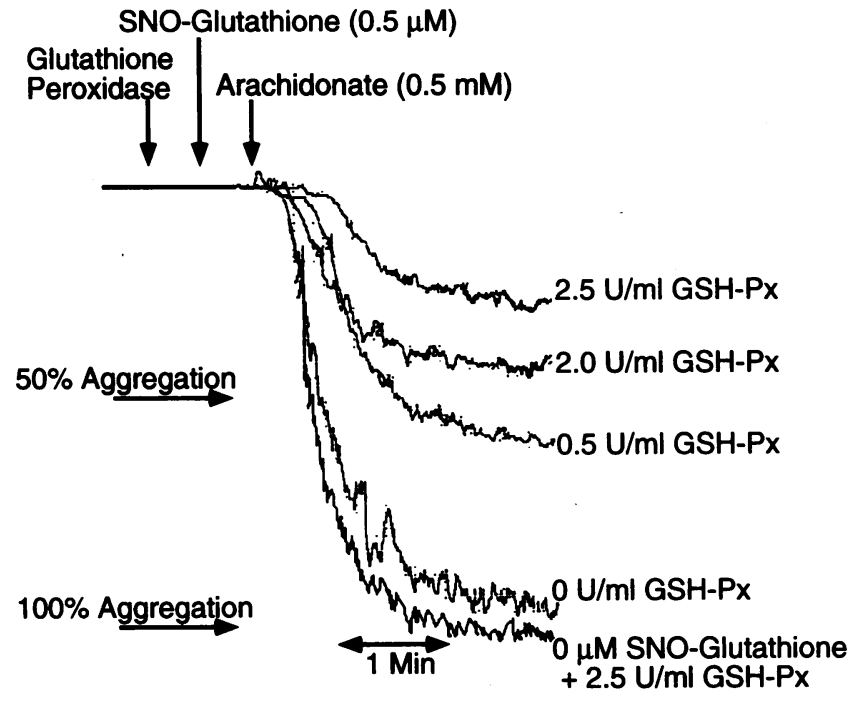

Figure 1. Effect of GSH-Px on platelet aggregation. GSH-Px was added $(0.5-2.5 \mathrm{U} / \mathrm{ml})$ to stirred PRP for $30 \mathrm{~s}$, followed by the addition of a subthreshold concentration of SNO-Glu $(0.5 \mu \mathrm{M})$. Platelet aggregation was induced by the addition of $0.5 \mathrm{mM}$ arachidonate. With increasing concentrations of GSH-Px, there is a dose-dependent augmentation of the inhibitory effects of SNO-Glu on platelet aggregation response. The addition of $2.5 \mathrm{U} / \mathrm{ml}$ GSH-Px in the absence of SNO-Glu does not alter the baseline platelet aggregation response.

using NADPH. Hydroperoxide reduction was followed by the decrease in absorbance at $340 \mathrm{~nm}(20)$. Activity was evaluated using either GSH, SNO-Glu, L-cysteinyl-glycine, L-cysteine, or D,L-homocysteine as the cosubstrate for GSH-Px.

Measurement of S-nitrosothiols. SNO-Cys and SNO-Glu were measured by HPLC using a C18 reverse-phase column coupled to an electrochemical detector with a dual $\mathrm{Au} / \mathrm{Hg}$ electrode set at both oxidizing $(+0.15 \mathrm{~V})$ and reducing $(-0.15 \mathrm{~V})$ potentials versus a $\mathrm{Ag} / \mathrm{AgCl}$ reference electrode (Bioanalytical Systems, West Lafayette, IN). This technique can reliably detect S-nitrosothiols in the nanomolar range (19). The working electrodes were configured in series with the reducing electrode upstream. Using this arrangement, S-nitrosothiols are detected at both electrodes, first as S-nitrosothiols at the reducing electrode and then as reduced thiol (the product of upstream reduction) at the downstream oxidizing electrode. S-nitrosothiols were identified and quantitated by comparison with authentic standards.

Statistics. All data are presented as means \pm SEM. Paired samples were compared by Student's $t$ test; values of $P<0.05$ are considered significant. Groups of data were tested by ANOVA and, if significant, were further evaluated by the Newman-Keuls test.

\section{Results}

GSH-Px, but not SOD or catalase, increases SNO-Glu-mediated attenuation of platelet aggregation. Increasing concentrations $(0.1-20 \mathrm{U} / \mathrm{ml})$ of GSH-Px, SOD, or catalase were added to PRP followed by stirring for $30 \mathrm{~s}$ after which a subthreshold concentration of SNO-Glu $(0.4-0.6 \mu \mathrm{M})$ was added. This subthreshold concentration, without enzyme, still allowed for $>90 \%$ platelet aggregation after the addition of arachidonate (Fig. 1). GSH-Px, but not SOD or catalase, potentiated the inhibition of platelet aggregation by SNO-Glu (Figs. 1 and 2). This effect was observed at concentrations as low as $0.2 \mathrm{U} / \mathrm{ml}$ of GSH-Px, with an $\mathrm{IC}_{50}$ of $0.6 \mathrm{U} / \mathrm{ml}(P<0.05$ by ANOVA compared with incubations with SNO-Glu alone). SOD or cata- 


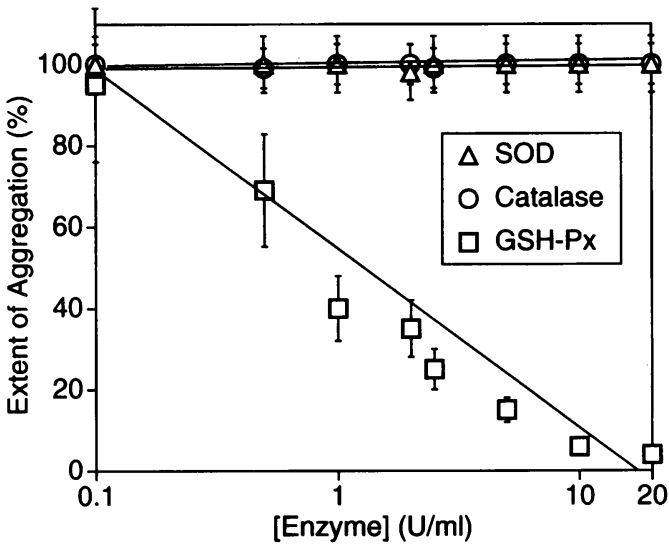

Figure 2. Effect of GSH-Px, SOD, or catalase inhibition of platelet aggregation by SNO-Glu. Increasing concentrations $(0.1-20 \mathrm{U} / \mathrm{ml})$ of GSH-Px, SOD, or catalase were added to PRP, followed by the addition of a subthreshold concentration of SNO-Glu $(0.5 \mu \mathrm{M})$. Aggregation was induced by the addition of $500 \mu \mathrm{M}$ arachidonate. GSH-Px ( $\square$ ), but not SOD $(\triangle)$ or catalase $(O)$, causes a dose-dependent augmentation of inhibition of platelet aggregation by SNO-Glu. (For GSH-Px, $P$ $<0.05$ by ANOVA compared with incubations with SNO-Glu alone; error bars represent SEM for $n=3$ experiments each performed in duplicate.)

lase alone or in combination (data not shown), at concentrations as great as $20-200 \mathrm{U} / \mathrm{ml}$, failed to inhibit platelet aggregation with SNO-Glu. Control studies, in which these enzymes were added in increasing concentrations without SNO-Glu or with GSH alone, showed no effect on platelet aggregation response (data not shown).

To study the effect of GSH-Px on inhibition of platelet aggregation by the direct NO donor, sodium nitroprusside, GSH-Px was added in increasing concentrations (0.1-20 $\mathrm{U} / \mathrm{ml}$ ) to stirred PRP $30 \mathrm{~s}$ before a subthreshold concentration of sodium nitroprusside $(0.2 \mu \mathrm{M})$. This concentration of nitroprusside, without enzyme, allowed for $>80 \%$ platelet aggregation. Unlike the S-nitrosothiols, sodium nitroprusside-induced inhibition of platelet aggregation was not altered by GSH-Px (data not shown).

Reversal of the potentiation of SNO-Glu-mediated inhibition of platelet aggregation by inhibitors of plasma GSH-Px. $\mathrm{Zn}^{2+}$ is a known inhibitor of endogenous plasma GSH-Px (21) that, unlike transition metal ions, does not promote free radical generation $(22,23)$. In addition, $\mathrm{ZnCl}_{2}$ inhibits the plasma but not the erythrocyte form of GSH-Px, excluding the possibility that the results obtained were due to erythrocyte contamination. 5 $\mathrm{mM} \mathrm{ZnCl}_{2}$ was added to PRP containing a concentration of SNO-Glu $(1.0 \mu \mathrm{M})$ that inhibited arachidonate-induced platelet aggregation by $>80 \%$. Addition of $\mathrm{ZnCl}_{2}$ led to a 2.1 -fold increase in platelet aggregation $(P<0.05)$ compared with control with SNO-Glu alone (Table I), whereas $\mathrm{ZnCl}_{2}$ in the absence of SNO-Glu did not alter platelet aggregation response (data not shown).

To confirm these results, $\beta$-mercaptosuccinic acid ( $33 \mu \mathrm{M})$, a specific inhibitor of selenium-dependent GSH-Px ( IC $_{50} 33$ $\mu \mathrm{M})(24)$, was added to PRP followed by SNO-Glu $(1.0 \mu \mathrm{M})$ and arachidonate. As shown in Table I, a 2.3-fold increase in platelet aggregation was observed under these conditions compared with control without inhibitor $(P<0.05)$.

Differential inhibition of platelet aggregation by S-nitro-
Table I. The Effect of GSH-Px Inhibitors on Platelet Inhibition by SNO-Glu in Plasma

\begin{tabular}{lc}
\hline & $\begin{array}{c}\text { Extent of } \\
\text { aggregation }\end{array}$ \\
\hline & $\%$ \\
SNO-Glu $(1 \mu \mathrm{M})$ & $18.0 \pm 3.2$ \\
SNO-Glu $(1 \mu \mathrm{M})+\mathrm{ZnCl}_{2}(5 \mathrm{mM})$ & $38.6 \pm 4.1$ \\
SNO-Glu $(1 \mu \mathrm{M})+\beta$-mercaptosuccinic acid $(33 \mu \mathrm{M})$ & $42.2 \pm 4.8$
\end{tabular}

The addition of $\mathrm{ZnCl}_{2}$ or $\beta$-mercaptosuccinic acid to PRP containing a concentration of SNO-Glu that inhibited platelet aggregation by $>80 \%$ led to a decrease in SNO-Glu-induced platelet inhibition. $(P<0.05$ for both $\mathrm{ZnCl}_{2}$ and $\beta$-mercaptosuccinic acid compared with SNO-Glu alone; for $n=3$ experiments each performed in duplicate.)

sothiols and GSH-Px. Increasing concentrations of GSH-Px (0$10 \mathrm{U} / \mathrm{ml}$ ) were added to PRP $30 \mathrm{~s}$ before the addition of subthreshold concentrations of either SNO-Glu $(0.5 \mu \mathrm{M})$ or SNOCys $(0.1 \mu \mathrm{M})$. The extent of platelet aggregation was compared at each concentration of GSH-Px after aggregation was induced by arachidonate. As shown in Fig. 3, inhibition of platelet aggregation by both SNO-Glu and SNO-Cys is potentiated by GSHPx; however, there is a rightward shift in dose dependency for SNO-Cys compared with SNO-Glu, with an $\mathrm{IC}_{50}$ of $3.2 \mathrm{U} / \mathrm{ml}$ versus $0.6 \mathrm{U} / \mathrm{ml}$, respectively ( $P<0.05$ by ANOVA).

$L O O H$ reverses the inhibition of platelet aggregation by SNO-Glu and GSH-PX. Increasing concentrations (0-4 $\mu \mathrm{M})$ of 12(S) HpETE or 5(S)HpETE were added to PRP $30 \mathrm{~s}$ before the addition of $1 \mathrm{U} / \mathrm{ml} \mathrm{GSH}-\mathrm{Px}$ in the presence of a concentration of SNO-Glu that caused 50\% inhibition of platelet aggregation. In the presence of SNO-Glu, the normal inhibitory effect of HpETEs was not seen. Instead, 12(S)HpETE alone, in a dose-dependent manner, attenuated inhibition of platelet aggregation by SNO-Glu, and this effect was partially reversed by

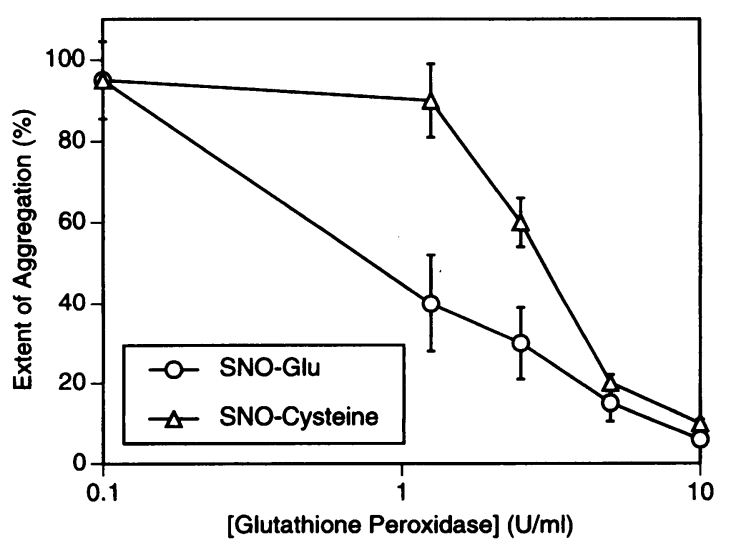

Figure 3. Effects of SNO-Glu and SNO-Cys on platelet aggregation in plasma. Extent of aggregation was compared after the addition of increasing concentrations of GSH-Px $(0-10 \mathrm{U} / \mathrm{ml})$ and subthreshold concentration of either SNO-Glu $(0.5 \mu \mathrm{M})(0)$ or SNO-Cys $(0.1 \mu \mathrm{M})(\Delta)$. $(P<0.05$ by ANOVA for SNO-Glu compared with SNO-Cys for GSH$\mathrm{Px} \leq 5 \mathrm{U} / \mathrm{ml}$; error bars represent SEM for $n=3$ experiments each performed in duplicate.) 


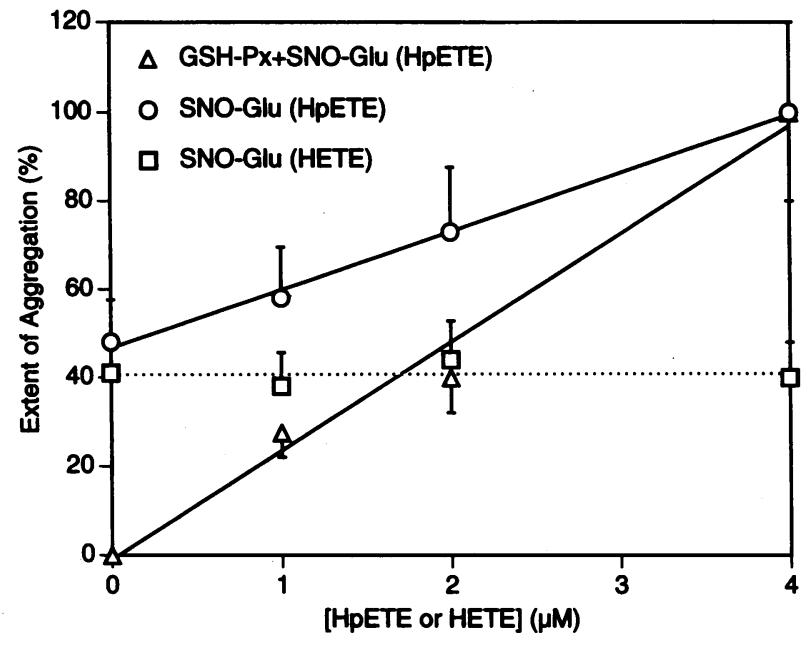

Figure 4. Effect of $\mathrm{LOOH}$ on the inhibition of platelet aggregation by SNO-Glu and GSH-Px. Increasing concentrations of 12(S)HpETE (0 $4 \mu \mathrm{M}$ ) or 12(S)HETE were added to PRP followed by the addition of threshold concentrations of SNO-Glu $(0.5 \mu \mathrm{M})$. HpETE samples were incubated in the presence and absence of $1 \mathrm{U} / \mathrm{ml} \mathrm{GSH}-\mathrm{Px}$. Increasing concentrations of $\mathrm{HpETE}(\mathrm{O})$ caused a dose-dependent increase in platelet aggregation, and this effect was attenuated by the addition of 1 U $/ \mathrm{ml} \mathrm{GSH-Px}(\Delta)$. Increasing concentrations of HETE alone did not alter platelet aggregation $(\square) .(P<0.05$ for SNO-Glu compared with SNO-Glu with GSH-Px for concentrations $\leq 2 \mu \mathrm{M}$ HpETE; error bars represent SEM for $n=3$ experiments each performed in duplicate.)

the addition of $1 \mathrm{U} / \mathrm{ml} \mathrm{GSH}-\mathrm{Px}$ (Fig. 4). Identical results were obtained with 5(S)HpETE (data not shown) but not 12(S)HETE (Fig. 4) or 5(S)HETE (data not shown). These data indicate that $\mathrm{LOOH}$, but not their corresponding alcohols, attenuate SNO-Glu-induced platelet inhibition and that this effect is modulated by GSH-Px.

Incubations with SNO-Glu, LOOH, and GSH-Px alter platelet cGMP levels. Incubations were conducted as previously described with PRP in the presence or absence of SNO-Glu, GSHPx, or HpETE. Before aggregation, the platelet proteins were precipitated with TCA, and the protein-free supernatant was analyzed for cGMP levels, a measure of the effects of NO exposure on platelet function. Compared with PRP alone (2.24 $\mathrm{pmol} / 10^{8}$ platelet), there was a significant increase in cGMP levels with the addition of $5 \mu \mathrm{M}$ SNO-Glu $\left(5.16 \mathrm{pmol} / 10^{8}\right.$ platelets) $(P<0.05)$ (Fig. 5). Incubation of PRP with either $5 \mathrm{U} / \mathrm{ml}$ GSH-Px or $5 \mu \mathrm{M}$ 12(S)HpETE did not significantly alter basal cGMP levels $\left(2.51\right.$ and 2.21 pmol/ $/ 10^{8}$ platelets, respectively). Incubation with 5 or $10 \mu \mathrm{M}$ HpETE and $5 \mu \mathrm{M}$ SNO-Glu significantly decreased cGMP levels compared with SNO-Glu alone (Fig. 5) $(P<0.05)$. Addition of $5 \mathrm{U} / \mathrm{ml}$ GSHPx restored the effects of SNO-Glu despite the presence of 5 $\mu \mathrm{M}$ HpETE (Fig. 5) $(P<0.05$ for SNO-Glu and HpETE compared with SNO-Glu, HpETE, and GSH-Px). Thus, the increases in platelet cGMP levels correlate with the extent of inhibition of platelet aggregation.

Cosubstrate affects GSH-Px activity. The activity of GSHPx $(4 \mathrm{U} / \mathrm{ml})$ with $200 \mu \mathrm{M} \mathrm{H}_{2} \mathrm{O}_{2}$ as substrate was measured using a coupled spectrophotometric assay. GSH, SNO-Glu, Lcysteinyl-glycine, D,L-homocysteine, L-cysteine, and SNO-Cys were used as potential cosubstrates over a range of concentrations $(0.125-1.25 \mathrm{mM})$. GSH-Px activity was measurable only

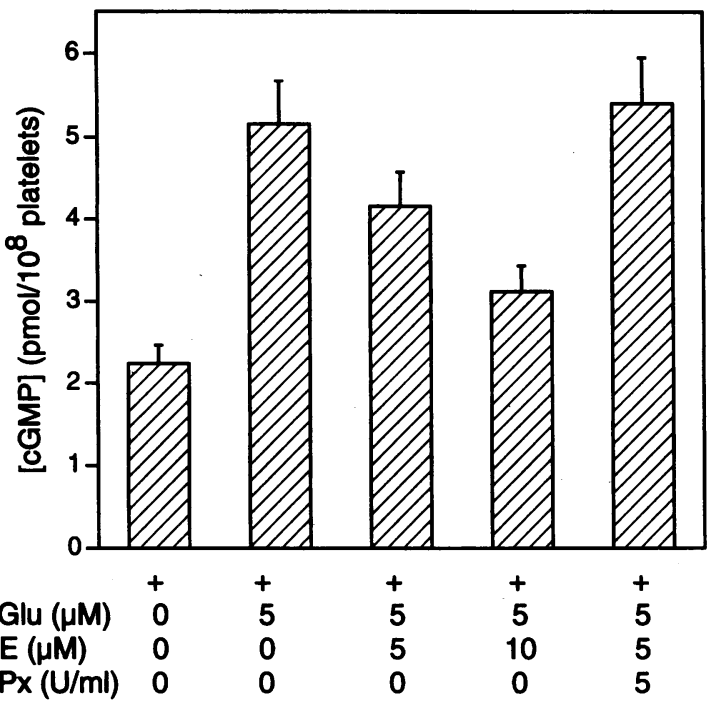

Figure 5. Effect of SNO-Glu, GSH-Px, and 12(S)HpETE on platelet cGMP levels. Incubation of PRP with 5 or $10 \mu \mathrm{M}$ HpETE and $5 \mu \mathrm{M}$ SNO-Glu significantly decreased cGMP levels compared with the addition of SNO-Glu alone $(P<0.05)$. Addition of $5 \mathrm{U} / \mathrm{ml}$ GSH-Px restored the effects of SNO-Glu despite the presence of $5 \mu \mathrm{M}$ HpETE $(P<0.05$ for SNO-Glu and HpETE compared with SNO-Glu, HpETE, and GSH$\mathrm{Px})$. Concentrations of cGMP are expressed as pmol $/ 10^{8}$ platelets. (Error bars represent SEM for $n=3$ experiments each performed in duplicate.)

with GSH and SNO-Glu as cosubstrates (Fig. $6 \mathrm{~A}$ ). Interestingly, SNO-Glu, which has not been previously described as a cosubstrate for GSH-Px, had virtually identical activity as GSH, with a $K_{\mathrm{m}}$ of $5.2 \mathrm{mM}$ for GSH and $5.4 \mathrm{mM}$ for SNO-Glu (Fig. $6 \mathrm{~B})$ and a $V_{\max }$ of $0.146 \Delta \mathrm{Abs} / \mathrm{min}$ for $\mathrm{GSH}$ and 0.167 $\triangle \mathrm{Abs} / \mathrm{min}$ for SNO-Glu.

GSH-Px increases transnitrosation from SNO-Glu to serum albumin. SNO-Glu ( $50 \mu \mathrm{M})$ was incubated with serum albumin $(50 \mu \mathrm{M})$ and $\mathrm{H}_{2} \mathrm{O}_{2}(200 \mu \mathrm{M})$ in PBS at room temperature, and the amount of S-nitroso-albumin formed was determined both in the presence and absence of GSH-Px. In the presence of GSH-Px there was an increased rate of loss of SNO-Glu over the 3-h incubation period (Fig. $7 \mathrm{~A}$ ). There was a commensurate increase in the rate of formation of S-nitroso-albumin, suggesting an increase in transnitrosation from SNO-Glu to serum albumin by GSH-Px (Fig. 7 B). GSH-Px, however, did not facilitate the transfer of NO from S-nitroso-albumin to GSH (data not shown).

Transnitrosation from SNO-Cys to GSH. The potentiation by GSH-Px of platelet inhibition with SNO-Cys may be a consequence of transnitrosation from SNO-Cys to endogenous (plasmatic) GSH. As shown above (Fig. 3), the effects of GSH-Px on S-nitrosothiol-mediated inhibition of platelet aggregation are less marked for SNO-Cys than SNO-Glu. This observation may be explained by the partial transfer of NO from SNOCys to GSH present in plasma forming SNO-Glu. Although transnitrosation reactions between serum albumin and low-molecular-weight thiols have been documented (24) and are suggested by the above data (Fig. 7), transnitrosation reactions between low-molecular-weight thiols have never been directly demonstrated. SNO-Cys was incubated with GSH in physiological buffer, and formation of SNO-Glu was measured by HPLC with electrochemical detection. The incubation of equimolar 

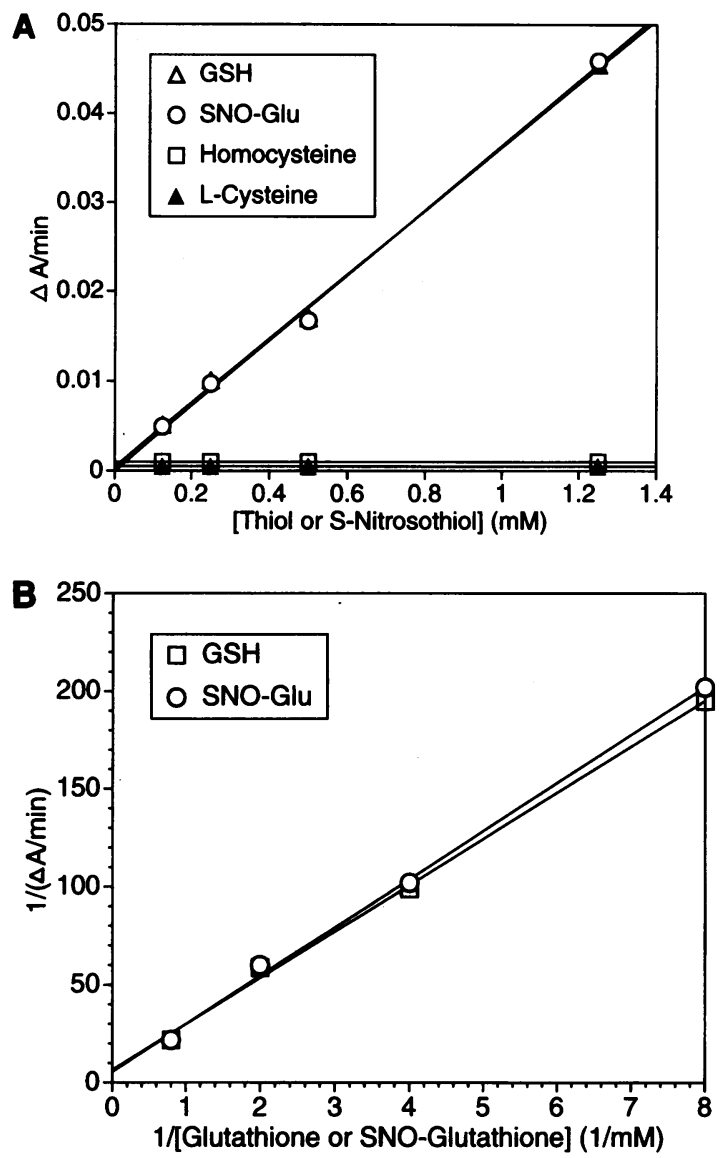

Figure 6. (A) Effect of cosubstrate on GSH-Px activity. The activity of GSH-Px $(4 \mathrm{U} / \mathrm{ml})$ was measured by a spectophotometric coupled assay using $\mathrm{H}_{2} \mathrm{O}_{2}(200 \mu \mathrm{M})$ and either $\mathrm{GSH}(\Delta)$, D,L-homocysteine $(\square)$, L-cysteine $(\Delta)$, or SNO-Glu $(O)$ as cosubstrates. Increasing concentrations of GSH or SNO-Glu $(0.125-1.25 \mathrm{mM})$ led to an increase in activity with similar rates for both GSH and SNO-Glu. D,L-homocysteine and L-cysteine had no activity in this assay. $(B)$ SNO-Glu $(O)$ had similar activity to GSH ( $\square$ ) as a cosubstrate for GSH-Px. Double reciprocal plots reveal a $K_{\mathrm{m}}$ of $5.2 \mathrm{mM}$ for GSH and $5.4 \mathrm{mM}$ for SNOGlu and a calculated $V_{\max }$ of $0.146 \Delta \mathrm{Abs} / \mathrm{min}$ for GSH and 0.167 $\Delta \mathrm{Abs} / \mathrm{min}$ for SNO-Glu.

concentrations ( $50 \mu \mathrm{M})$ of SNO-Cys and GSH at room temperature for $3 \mathrm{~min}$ led to the formation of $36.0 \pm 9.2 \mu \mathrm{M}$ of SNOGlu and a concomitant decrease in the concentration of SNOCys by $30.0 \pm 5.0 \mu \mathrm{M}$. These data indicate that the addition of exogenous SNO-Cys leads to the transfer of NO to another physiological low-molecular-weight thiol, forming SNO-Glu.

GSH-Px decreases SNO-Glu concentrations. Using the HPLC-electrochemical detection method, the concentration of SNO-Glu was measured after incubation with washed platelets and $\mathrm{H}_{2} \mathrm{O}_{2}$, both in the presence and absence of GSH-Px. SNOGlu $(50 \mu \mathrm{M})$ was incubated for $1 \mathrm{~min}$ with $5 \mathrm{U} / \mathrm{ml} \mathrm{GSH}-\mathrm{Px}$, a concentration that potentiated platelet inhibition by SNO-Glu. There was a decrease in the concentration of SNO-Glu from $50.3 \pm 0.5 \mu \mathrm{M}$ without GSH-Px to $25.9 \pm 5.4 \mu \mathrm{M}$ in the presence of GSH-Px (Table II) $(P<0.05$ for SNO-Glu with GSH-Px compared with SNO-Glu without GSH-Px for $n=3$ experiments). These data suggest that potentiation of S-nitrosothiolinduced inhibition of platelet aggregation by GSH-Px is related
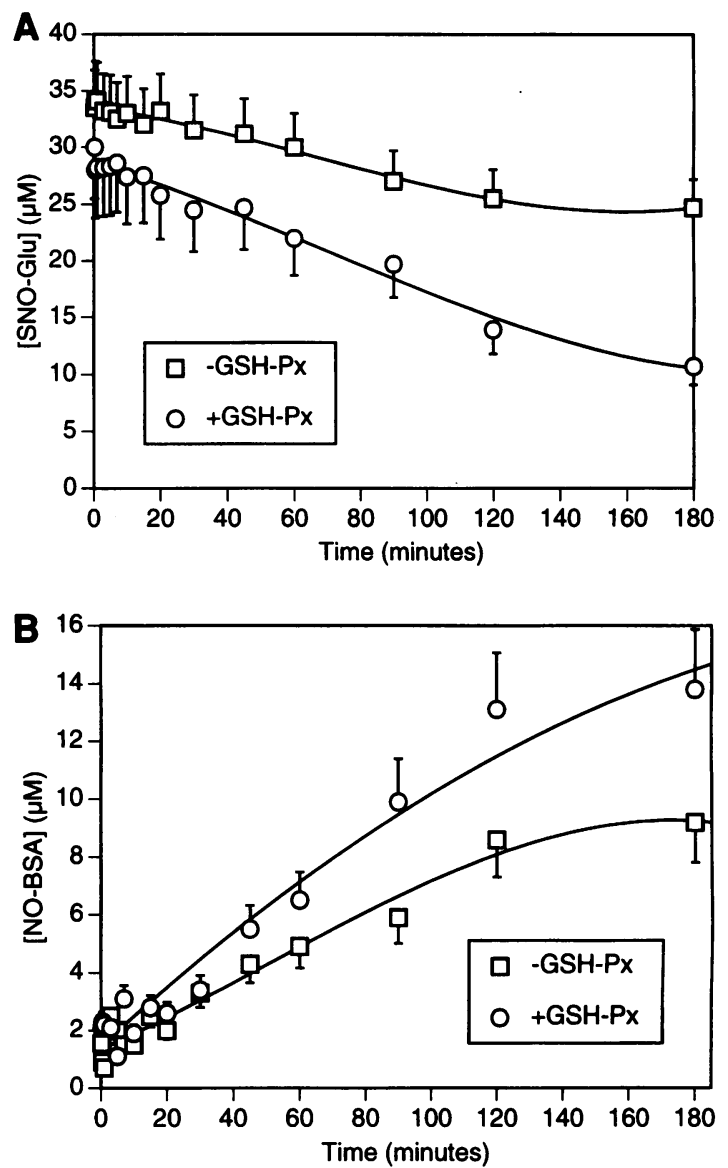

Figure 7. Effect of GSH-Px on transnitrosation from SNO-Glu to albumin. SNO-Glu ( $50 \mu \mathrm{M})$, serum albumin $(50 \mu \mathrm{M})$, and $\mathrm{H}_{2} \mathrm{O}_{2}(200 \mu \mathrm{M})$ were incubated in PBS and the amount of S-nitroso-albumin formed was determined both in the presence and absence of $5 \mathrm{U} / \mathrm{ml}$ GSH-Px. (A) Effect of GSH-Px on loss of SNO-Glu. In the presence of GSHPx $(O)$, increased loss of SNO-Glu is detected compared with the reaction without the enzyme $(\square) .(P<0.05$ by ANOVA comparing reactions with and without GSH; error bars represent SEM for $n=3$ experiments each performed in duplicate.) ( $B$ ) Effect of GSH-Px on S-nitrosoBSA formation. In the presence of GSH-Px (O) there is increased formation of S-nitroso-BSA compared with the identical incubation without the enzyme ( $\square) .(P<0.05$ by ANOVA; error bars represent SEM for $n=3$ experiments each performed in duplicate.)

to the enzyme's use of SNO-Glu as a cosubstrate coupled with the release of NO.

\section{Discussion}

Previous studies have demonstrated that $\mathrm{LOOH}$ and NO critically affect platelet function and that GSH-Px is the major hydroperoxide-reducing agent in plasma $(10,16)$. Therefore, in the present study we investigated the effects of GSH-Px on platelet inhibition by S-nitrosothiol. We found that GSH-Px potentiates S-nitrosothiol-induced inhibition of platelet aggregation in a dose-dependent manner with a concomitant decrease in the concentration of SNO-Glu. These effects were not seen with sodium nitroprusside, a direct NO donor, and were dependent on the type of S-nitrosothiol used. In addition, SNO-Glu was found to be equally active as GSH as a cosubstrate for GSH-Px, and increased transnitrosation from SNO-Glu to serum 
Table II. Utilization of SNO-Glu by GSH-Px in the Reduction of $\mathrm{H}_{2} \mathrm{O}_{2}$

\begin{tabular}{lc}
\hline & [SNO-Glu] \\
\hline & $\mu M$ \\
Preincubation & $50.3 \pm 0.5$ \\
Incubation without GSH-Px & $50.0 \pm 2.3$ \\
Incubation with GSH-Px (5 U/ml) & $25.9 \pm 5.4$ \\
\hline
\end{tabular}

SNO-Glu $(50 \mu \mathrm{M})$ was incubated with GSH-Px and $\mathrm{H}_{2} \mathrm{O}_{2}$ in PBS for $1 \mathrm{~min}$, and the concentration of SNO-Glu was measured by HPLC and electrochemical detection. There is a $49.5 \%$ decrease in the concentration of SNO-Glu after incubation with GSH-Px accompanying the reduction of $\mathrm{H}_{2} \mathrm{O}_{2}(P<0.05$ for SNO-Glu with GSH-Px compared with SNO-Glu alone for $n=3$ experiments).

albumin was observed in the presence of GSH-Px. These data suggest that GSH-Px is important in NO regulation via metabolism of the naturally occurring SNO-Glu.

Although a role for GSH-Px in NO regulation has not been previously described, such an association is consistent with studies on selenium, the trace element necessary for GSH-Px activity. Dietary selenium supplementation, at levels far below toxicity, correlates with increases in GSH-Px activity in normal individuals, as well as a prolongation in bleeding time (25). Platelets of selenium-deficient rats demonstrate an increased aggregation response (26). Deficient whole-blood selenium levels correlate with lower platelet GSH-Px activity without significant differences in vitamin $E$ levels or compensatory increases in SOD activity (27). Selenium deficiency in children correlates with decreased GSH-Px activity in plasma, also without any compensatory increase in SOD activity.

GSH-Px is responsible for most of the hydroperoxide-reducing activity in human plasma $(10,16)$ and thus plays a role in the protection against oxidative damage. Our data suggest that LOOH can interact with and inactivate NO. Normally, no LOOH can be detected in plasma $(<30 \mathrm{nM})(28)$, but a local and/or transient increase may occur during oxidant stress or in the local milieu of the platelet aggregate. Platelet aggregation and subsequent stimulation of the arachidonic acid pathway in platelets leads to the generation of HpETEs and HETEs by the lipoxygenase pathway (29). As we have shown, 12(S)HpETE, but not 12(S)HETE, reverses the inhibitory effects of NO on platelet aggregation. Therefore, $\mathrm{LOOH}$ formed during the activation of platelets may react with NO, promoting further platelet activation. GSH-Px, through catalysis of reduction of $\mathrm{LOOH}$ normally produced during platelet activation, thus has potential antithrombotic properties.

In addition to the antioxidant action of GSH-Px and the prevention of a reaction between $\mathrm{LOOH}$ and NO, the preservation of SNO-Glu-induced inhibition of platelet aggregation could result from the interaction of the S-nitrosothiol itself with the enzyme. Although GSH-Px has been reported to be specific for GSH, our experiments show that the S-nitrosated form of GSH is an equivalently effective cosubstrate. The observation that SNO-Glu concentration decreases in the presence of GSHPx suggests that GSH-Px increases the availability of NO from SNO-Glu or converts it to a form that can more readily interact with platelets. However, SNO-Cys was not a substrate for GSH$\mathrm{Px}$, and the effects of GSH-Px on platelet function were greater in the presence of SNO-Glu than SNO-Cys. The finding that GSH-Px potentiates platelet inhibition by SNO-Cys indicates that NO is transferred from SNO-Cys to plasmatic GSH, which then acts as a substrate for GSH-Px. Indeed, we observed that SNO-Glu is formed from SNO-Cys in the presence of GSH. Therefore, the transient formation of SNO-Glu may be necessary for the enzyme to support platelet inhibition by NO, regardless of the S-nitrosothiol initially present. This effect of GSHPx on SNO-Glu metabolism may also explain why GSH-Px did not potentiate platelet inhibition by sodium nitroprusside, as sodium nitroprusside does not support formation of SNO-Glu from GSH. In addition, although GSH-Px increased the transnitrosation from SNO-Glu to serum albumin, it did not facilitate the transfer of NO from S-nitroso-albumin to GSH. Although the precise mechanism for transnitrosation remains unknown, these experiments suggest that in the presence of GSH-Px, SNO-Glu may react with $\mathrm{LOOH}$ or $\mathrm{H}_{2} \mathrm{O}_{2}$ forming oxidized glutathione, the lipid hydroxide or $\mathrm{H}_{2} \mathrm{O}$, and $\mathrm{N}_{2} \mathrm{O}_{3}$, a known nitrosating species.

The $K_{\mathrm{m}}$ of GSH-Px for reduced GSH in plasma (4.3 mM) is several orders of magnitude higher than the plasma concentrations of GSH $(0.34-5 \mu \mathrm{M})(30,31)$. However, the enzyme has a low $K_{\mathrm{m}}$ value for organic hydroperoxides $(2.3 \mu \mathrm{M})$ as well as a high catalytic constant $\left(k_{\text {cat }}\right)$, which in the setting of decreased substrate concentrations can allow for significant biological activity. In freshly obtained human plasma, Px activity can be detected for phosphatidylcholyl, free fatty acyl, and cholesteryl hydroperoxides without the addition of an exogenous reduced cosubstrate $(32,33)$. Thus, plasma GSH-Px appears to be a highly efficient enzyme for the reduction of organic hydroperoxides, even at relatively low plasma concentrations of GSH. GSH-Px may have a physiological role in the control of circulating fatty acyl hydroperoxides as well as phospholipid and cholesteryl hydroperoxides, species known to be elevated in atherosclerotic lesions $(34,35)$. Intracellular selenium-dependent GSH-Px has also been shown to play a significant role in the enzymatic reduction of hydroperoxides generated by platelets (36). Unstimulated platelets maintain an intracellular GSH concentration of 3-5 $\mathrm{mM}$. Upon activation, there is a transient $80 \%$ decrease in GSH levels due to consumption by GSH-Px. Although the relevance of these observations for in vivo responses remains to be demonstrated, it is conceivable that in the setting of inflammatory reactions, where large quantities of $\mathrm{LOOH}$ and $\mathrm{H}_{2} \mathrm{O}_{2}$ are formed, GSH-Px is depleted and the reducing capacity of platelet GSH-Px is overwhelmed. As a consequence, increased quantities of $\mathrm{LOOH}$ would be available to interact with NO, potentially modulating platelet responses.

In summary, we have shown that GSH-Px has a potent effect on S-nitrosothial-induced inhibition of platelet aggregation. Thus, this enzyme may have two functions: reduction of $\mathrm{LOOH}$, thereby preventing inactivation of NO; and metabolism of SNOGlu, thereby liberating NO and/or supporting further transnitrosation reactions. This study, therefore, shows that GSH-Px regulates the availability of NO, possibly modulating its effects on platelet activation and platelet-vessel wall interactions.

\section{Acknowledgments}

We thank Ms. Anne Marie Ward for her expert technical assistance and Ms. Stephanie Tribuna for her expert secretarial assistance. We also 
thank Dr. Peter Brecher for helpful suggestion upon review of the manuscript.

This work was supported in part by National Institutes of Health (NIH) grants HL-48743 and HL-53919, a Merit Review Award from the U.S. Veterans Administration, and a grant from Nitro Med, Inc. Dr. Freedman is the recipient of a Fellowship Award from the Massachusetts Affiliate of the American Heart Association, Dr. Frei is the recipient of an NIH FIRST Award (HL-49954), and Dr. Loscalzo is the recipient of a Research Career Development Award from the NIH (HL-02273).

\section{References}

1. Azuma, H., M. Ishikawa, and S. Sekizaki. 1986. Endothelium-dependent inhibition of platelet aggregation. Br. J. Pharmacol. 88:411-415.

2. Radomski, M. W., R. M. J. Palmer, and S. Moncada. 1987. Comparative pharmacology of endothelium-derived relaxing factor, nitric oxide and prostacyclin in platelets. Br. J. Pharmacol. 92:181-187.

3. Radomski, M. W., R. M. J. Palmer, and S. Moncada. 1987. Endogenous nitric oxide inhibits human platelet adhesion to vascular endothelium. Lancet. 2:1057-1058.

4. Ignarro, L. J., R. E. Byrns, G. M. Buga, and K. S. Wood. 1987. Endotheliumderived relaxing factor from pulmonary artery and vein possesses pharmacologic and chemical properties identical to those of nitric oxide radical. Circ. Res. 61:866-879.

5. Shultz, P. J., and L. Raij. 1992. Endogenously synthesized nitric oxide prevents endotoxin-induced glomerular thrombosis. J. Clin. Invest. 90:17181725.

6. Radomski, M. W., M. J. Palmer, and S. Moncada. 1987. The role of nitric oxide and cGMP in platelet adhesion to vascular endothelium. Biochem. Biophys. Res. Commun. 148:1482-1489.

7. Rubbo, H., R. Radi, M. Trujillo, R. Telleri, B. Kalyanaraman, S. Barnes, M. Kirk, and B. A. Freeman. 1994. Nitric oxide regulation of superoxide and peroxynitrite-dependent lipid peroxidation. J. Biol. Chem. 269:26066-26075.

8. Avissar, N., J. C. Whitin, P. Z. Annen, I. S. Palmer, and H. J. Cohen. 1989. Antihuman plasma glutathione peroxidase antibodies: immunologic investigations to determine plasma glutathione peroxidase protein and selenium content in plasma. Blood. 73:318-323.

9. Aharony, D., J. B. Smith, and M. J. Silver. 1982. Regulation of arachidonateinduced platelet aggregation by the lipoxygenase product, 12-hydroperoxyeicosatetraenoic acid. Biochim. Biophys. Acta. 718:193-200.

10. Maddipati, K. R., C. Gasparski, and L. J. Marnett. 1987. Characterization of the hydroperoxide-reducing activity of human plasma. Arch. Biochem. Biophys. 254:9-17.

11. Buczynski, A., B. Wachowicz, K. Dedziora-Kornatowska, W. Tkaczewski, and J. Kedziora. 1993. Changes in antioxidant enzyme activities, aggregability and malonaldialdehyde concentration in blood platelets from patients with coronary heart disease. Atherosclerosis 100:223-228.

12. Salonen, J. T., G. Alfthan, J. K. Huttunen, J. Pikkarainen, and P. Puska. 1982. Association between cardiovascular death and myocardial infarction and serum selenium in a matched-pair longitudinal study. Lancet. 2:175-179.

13. Gabrielsen, B. O., and J. Opstvedt. 1980. Availability of selenium in fish meal in comparison with soya bean meal, corn glutin mean and selenomethionine reletive to selenium in sodium selenite for restoring glutathione peroxidase activity in selenium-depleted chicks. J. Nutr. 10:1096-1100.

14. Born, G. V., and M. J. Cross. 1963. The aggregation of blood platelets. J. Physiol. (Lond.). 168:178-195.

15. Stamler, J. S., D. I. Simon, J. A. Osborne, M. E. Mullins, O. Jaraki, T. Michel, D. J. Singel, and J. Loscalzo. 1992. S-nitrosylation of proteins with nitric oxide: synthesis and characterization of biologically active compounds. Proc. Natl. Acad. Sci. USA. 89:444-448.

16. Maddipati, K. R., and L. J. Marnett. 1987. Characterization of the major hydroperoxide-reducing activity of human plasma. J. Biol. Chem. 262:1739817403.

17. Wendel, A. 1980. Enzymatic Basis of Detoxification vol. I. W. B. Jacoby, editor. Academic Press, New York. 333-353.

18. Saville, B. 1958. A scheme for the colorimetric determination of microgram amounts of thiols. Analyst. 83:670-672.

19. Scharfstein, J. S., J. R. Keaney, A. Slivka, G. N. Welch, J. A. Vita, J. S. Stamler, and J. Loscalzo. 1994. In vivo transfer of NO between a plasma proteinbound reservoir and low-molecular-weight thiols. J. Clin. Invest. 94:1432-1439.

20. Pascual, P., E. Martinez-Lara, J. A. Barcena, J. Lopez-Barea, and F. Toribio. 1992. Direct assay of glutathione peroxidase activity using high-performance capillary electrophoresis. J. Chromatogr. 581:49-56.

21. Takahashi, K., N. Avissar, J. Whitin, and H. Cohen. 1987. Purification and characterization of human plasma glutathione peroxidase: a selenoglycoprotein distinct from the known cellular enzyme. Arch. Biochem. Biophys. 256:677-686.

22. Wilkins, G. M., and D. S. Leake. 1994. The oxidation of low density lipoproteins by cells or iron is inhibited by zinc. FEBS (Fed. Eur. Biochem. Soc.) Lett. 341:259-262.

23. Heinecke, J. W., H. Rosen, and A. Chait. 1984. Iron and copper promote modification of low density lipoprotein by human arterial smooth muscle cells in culture. J. Clin. Invest. 74:1890-1894.

24. Chaudiere, J., E. C. Wilhelmsen, and A. L. Tappel. 1984. Mechanism of selenium-glutathione peroxidase and its inhibition by mercaptocarboxylic acids and other mercaptans. J. Biol. Chem. 259:1043-1050.

25. Schiavon, R., G. E. Freeman, G. C. Guidi, G. Perona, M. Zatti, and V. V. Kakkar. 1984. Selenium enhances prostacyclin production by cultured endothelial cells: possible explanation for increased bleeding times in volunteers taking selenium as a dietary supplement. Thromb. Res. 34:389-396.

26. Schoene, N. W., V. C. Morris, O. A. Levander. 1986. Altered arachidonic acid metabolism in platelets and aortas from selenium-deficient rats. Nutr. Res. 6:75-83.

27. Wu, J., and G. L. Xu. 1987. Plasma selenium content, platelet glutathione peroxidase and superoxide dismutase activity of residents in Kashin-Beck disease affected area in China. J. Trace Elem. Electrolytes Health Dis. 1:39-43.

28. Frei, B., Y. Yamamoto, D. Niclas, and B. N. Ames. 1988. Evaluation of an isoluminol chemiluminescence assay for the detection of hydroperoxides in human blood plasma. Anal. Biochem. 175:120-130.

29. Jahn, B., and G. M. Hansch. 1990. Oxygen radical generation in human platelets: dependence on 12-lipoxygenase activity and on the glutathione cycle. Int. Arch. Allergy Appl. Immunol. 93:73-79.

30. Wendel, A., and P. Cikryt. 1980. The level and half-life of glutathione in human plasma. FEBS (Fed. Eur. Biochem. Soc.) Lett. 120:209-211.

31. Buhl, R., K. J. Holroyd, A. Mastrangeli, A. M. Cantin, H. A. Jaffe, F. B. Wells, C. Saltin, and R. G. Crystal. 1989. Systemic glutathione deficiency in symptom-free HIV-seropositive individuals. Lancet. ii:1294-1297.

32. Frei, B., R. Stocker, and B. N. Ames. 1988. Antioxidant defenses and lipid peroxidation in human blood plasma. Proc. Natl. Acad. Sci. USA. 85:97489752.

33. Terao, J., S. S. Shibata, and S. Matsushita. 1988. Selective quantification of arachidonic acid hydroperoxides and their hydroxy derivatives in reverse-phase high performance liquid chromatography. Anal. Biochem. 169:415-423.

34. Harland, H., J. Gilbert, and C. Brooks. 1973. Lipids of human atheroma. Biochim. Biophys. Acta. 316:378-385.

35. Mowri, H., H. Chinen, S. Ohkuma, and T. Takano. 1986. Peroxidized lipids isolated by HPLC from atherosclerotic aorta. Biochem. Int. 12:347-352.

36. Bryant, R. W., T. C. Simon, and J. M. Bailey. 1983. Hydroperoxy fatty acid formation in selenium deficient rat platelets: coupling of glutathione peroxidase to the lipoxygenase pathway. Biochem. Biophys. Res. Commun. 117:183-189. 